\title{
A CHARACTERIZATION OF SEQUENTIAL RATIONALIZABILITY
}

\author{
JOSE APESTEGUIA ${ }^{\dagger}$ AND MIGUEL A. BALLESTER ${ }^{\ddagger}$
}

\begin{abstract}
A choice function is sequentially rationalizable if there is an ordered collection of asymmetric binary relations that identifies the selected alternative in every choice problem. We propose a property, $\mathcal{F}$-consistency, and show that it characterizes the notion of sequential rationalizability. $\mathcal{F}$-consistency is a testable property that highlights the behavioral aspects implicit in sequentially rationalizable choice. Further, our characterization result provides a novel tool with which to study how other behavioral concepts are related to sequential rationalizability, and establish a priori unexpected implications. In particular, we show that the concept of rationalizability by game trees, which, in principle, had little to do with sequential rationalizability, is a refinement of the latter. Every choice function that is rationalizable by a game tree is also sequentially rationalizable. Finally, we show that some prominent voting mechanisms are also sequentially rationalizable.
\end{abstract}

Keywords: Individual rationality, Rationalizability, Consistency, Bounded rationality, Behavioral economics, Voting.

JEL classification numbers: B41, D01.

\section{INTRODUCTION}

The classic choice model in economics encompasses choice behaviors that are the result of maximizing a preference relation. When this is the case, any such choice behavior is typically said to be rationalizable. Over the last decades, however, the research has produced increasing amounts of evidence documenting systematic and predictable deviations from the notion of rationality implied in the above definition. Not surprisingly, these inconsistencies between theoretical models and applications have allowed a number of alternative rationalizability models to flourish in the literature. In general, these models adopt less strict notions of rationality. Some recent examples are:

Date: May, 2008.

We thank Paola Manzini, Marco Mariotti, Prasanta Pattanaik, Michael Richter, Karl Schlag Yongsheng $\mathrm{Xu}$, and Lin Zhou for valuable comments. The research was sponsored by the Spanish Commission of Science and Technology (SEJ2006-11510 and SEJ2005-01481/ECON) and the Barcelona Economics Program of CREA.

${ }^{\ddagger}$ Universitat Pompeu Fabra. E-mail: jose.apesteguia@upf .edu.

${ }_{\ddagger}^{\ddagger}$ Universitat Autonoma de Barcelona. E-mail: miguelangel.ballester@uab.es. 
- Rationalization by multiple rationales (Kalai, Rubinstein, and Spiegler, 2002): ${ }^{1}$ the decision-maker (DM) partitions the set of choice problems into different categories and applies different rationales to different categories.

- Rationalizability by game trees (Xu and Zhou, 2007): the choices of the DM are the equilibrium outcome of an extensive game with perfect information.

- Sequential Rationalizability (Manzini and Mariotti, 2007): the DM sequentially applies a collection of rationales in a fixed order.

It is the last of these approaches that concerns us here. The intuition of Manzini and Mariotti's notion of sequential rationalizability is very appealing from a behavioral perspective. It considers DMs that, when faced with a choice problem, apply a number of criteria in a fixed order of priority, gradually narrowing down the set of alternatives until one is identified as the choice. Notably, Manzini and Mariotti provide characterizations for those choice functions that are sequentially rationalizable by two rationales, named Rational Shortlist Methods (RSMs), and three rationales. ${ }^{2}$ That is, the work of Manzini and Mariotti allows us to identify choice behavior that can be sequentially rationalized by either two or by three rationales.

The question then arises of how to identify choice behavior that may be sequentially rationalizable, but only by means of more than three rationales. In this paper we propose a testable property of choice functions, $\mathcal{F}$-consistency, that turns out to be equivalent to the fact that a choice function $c$ is sequentially rationalizable. $\mathcal{F}$ consistency has a behavioral flavor and falls within the tradition of the properties that can be found in the choice-theoretic literature. $\mathcal{F}$-consistency suggests a particular behavioral procedure that DMs may follow. It evokes the existence of a two-stage procedure in which the DM first makes a preselection of alternatives following a rule requiring a great deal of behavioral consistency, and then makes the final choice.

Our characterization allows a better understanding of sequential rationalizability. Further, we show that this result opens up the possibility of linking sequential rationalizability with other rationalizability notions, and determining whether certain choice patterns are sequentially rationalizable. In particular, we first show that the notion of rationalizability by game trees, due to Xu and Zhou (2007), which, in principle, had little to do with sequential rationalizability, is a strict refinement of the latter. Our property can be used to show that every choice function that is rationalizable by game trees is in fact sequentially rationalizable, and a simple example shows that the reverse does not hold. We then show that agenda rationalizability, a rationalizability notion that we introduce here and that is rooted in certain models of choice by ordered elimination and also in voting mechanisms based on successive elimination,

\footnotetext{
${ }^{1}$ For the moment take a rationale as a preference relation. We will be more precise later.

${ }^{2}$ Salant and Rubinstein (2008) provide an alternative characterization of RSMs in the framework of a 'limited attention' model.
} 
is also sequentially rationalizable. The proofs of these two links with sequential rationalizability constitute one of the direct implications of our characterization result. Further, these two links suggest that the notion of sequential rationalizability may be of interest in more environments than initially expected.

The paper is organized as follows. Section 2 introduces the notation and the main definitions to be used thereafter. Section 3 contains the main result of the paper: the characterization result. Section 4 presents two applications of sequential rationalizability: (i) rationalizability by game trees and (ii) agenda rationalizability. Finally, Section 5 concludes.

\section{Basic Notation and Definitions}

Let $X$ be a finite set of $n \geq 2$ objects. ${ }^{3}$ We denote by $\mathcal{P}(X)$ the set of all nonempty subsets of $X$. A choice function $c$ on $\mathcal{P}(X)$ assigns to every $A \in \mathcal{P}(X)$ a unique element $c(A) \in A$.

Denote by $P$ an asymmetric binary relation on $X, P \subseteq X \times X$. That is, if $x P y$ for some $x, y$ in $X$, then it is not true that $y P x$. We will often refer to $P$ as a rationale. For any $A \in \mathcal{P}(X), M(A, P)$ denotes the set of maximal elements in $A$ with respect to $P$, that is $M(A, P)=\{x \in A: y P x$ for no $y \in A\}$. We will often write $M(A)$ to refer to $M(A, P)$.

The classic notion of rationalizability of a choice function $c$ deals with the issue of existence of a binary relation $P$ that explains choice behavior as the result of maximization. That is, a choice function $c$ is rationalizable if there is a binary relation $P$ such that, for any choice problem $A \in \mathcal{P}(X), c(A)=M(A) \cdot{ }^{4}$ A well-known result establishes that a choice function $c$ is rationalizable if and only if $c$ satisfies the Independence of Irrelevant Alternatives (IIA) property. ${ }^{5}$

Here we are interested in Manzini and Mariotti's sequential notion of rationalizability. A choice function $c$ is sequentially rationalizable by the ordered collection of rationales $\left\{P_{1}, \ldots, P_{K}\right\}$ if, for every choice problem $A$, the sequential application of the rationales in that fixed order identifies the selection of $c$. It is important to stress that the order of application of rationales is fixed across choice problems. In order to introduce the formal definition of sequential rationalizability, given an ordered collection of rationales $\left\{P_{1}, \ldots, P_{K}\right\}$, let $M_{0}(A)=A$ and define recursively for $j=1, \ldots, K$,

\footnotetext{
${ }^{3}$ Manzini and Mariotti consider not necessarily finite sets of objects. In order to avoid tedious technical details that add little to the understanding of sequential rationalizability, we chose to focus on the finite case. Details of suitable extensions to the general case can be provided upon request. Also, in relation to the next section, the setting of $\mathrm{Xu}$ and Zhou (2007) is finite.

${ }^{4}$ With a slight abuse of notation, we identify elements with sets containing only one element.

${ }^{5}$ IIA simply states that, if an alternative is chosen from a set, then it should also be chosen in every subset of the set that contains the alternative.
} 
$M_{j}(A)=M\left(M_{j-1}(A), P_{j}\right)=\left\{x \in M_{j-1}(A): y P_{j} x\right.$ for no $\left.y \in M_{j-1}(A)\right\}$.

Sequential Rationalizability: A choice function $c$ is sequentially rationalizable whenever there exists a non-empty ordered list $\left\{P_{1}, \ldots, P_{K}\right\}$ of asymmetric binary relations such that $c(A)=M_{K}(A)$ for all $A \in \mathcal{P}(X)$.

In a sequential rationalization of a choice function, successive application of the rationales eventually reduces the set of alternatives to a unique element, which is precisely the choice according to $c$.

\section{A Characterization of Sequential Rationalizability}

In this section we introduce a new property of choice functions and show that it is equivalent to the notion of sequential rationalizability. To this end, we first need to introduce some definitions. We say that a collection of choice problems $\mathcal{F} \subseteq \mathcal{P}(X)$ is a filter if: (i) for every set $A$ in $\mathcal{F}$, every non-empty subset of $A$ also belongs to the collection $\mathcal{F}$, and (ii) there exists at least one problem set $C \in \mathcal{F}$ with $|C| \geq 2$. Note that the collection of all choice problems $\mathcal{P}(X)$ is a filter.

We now introduce a choice correspondence, $\gamma_{\mathcal{G}}$ defined on an arbitrary domain described by a collection of problem sets $\mathcal{G} \subseteq \mathcal{P}(X)$, that assigns to every $A \in \mathcal{G}$ a non-empty set of elements $\gamma_{\mathcal{G}}(A) \subseteq A$. We say that the choice correspondence $\gamma_{\mathcal{G}}$ is non-trivial if it is not true that $\gamma_{\mathcal{G}}(A)=A$ for every $A \in \mathcal{G}$. $\gamma_{\mathcal{G}}$ satisfies the $\alpha$ property (or simply $\alpha$ ), if, for any $A, B \in \mathcal{G}$, if $x \in A \subseteq B$ and $x \in \gamma_{\mathcal{G}}(B)$, then $x \in \gamma_{\mathcal{G}}(A){ }^{6}$ $\gamma_{\mathcal{G}}$ satisfies Always Chosen $(\mathrm{AC})$ if for any $S \in \mathcal{G}$, if $\{x, y\} \in \mathcal{G}$ and $x \in \gamma_{\mathcal{G}}(\{x, y\})$ for all $y \in S \backslash\{x\}$, then $x \in \gamma_{\mathcal{G}}(S)$. We are now in a position to introduce the key behavioral property of choice behavior.

$\mathcal{F}$-Consistency: A choice function $c$ satisfies $\mathcal{F}$-consistency if, for every filter $\mathcal{F} \subseteq \mathcal{P}(X)$, there exists a choice correspondence $\gamma_{\mathcal{F}}$ such that $\gamma_{\mathcal{F}}$ is non-trivial, satisfies $\alpha$, AC, and $c(A)=c\left(\gamma_{\mathcal{F}}(A)\right)$ for every $A \in \mathcal{F}$.

$\mathcal{F}$-consistency suggests a two-stage procedure. In the first-stage, the correspondence $\gamma_{\mathcal{F}}$ makes a 'rational' preselection of alternatives, and then, in the second-stage, the final choice takes place. We speak of a 'rational' preselection of alternatives because $\mathcal{F}$-consistency imposes a great deal of structure on the choice correspondences $\gamma_{\mathcal{F}} . \mathcal{F}$-consistency imposes $\alpha$ and substitutes the classical property $\beta$ for AC. ${ }^{7}$ This means that we allow for some, but not all, violations of property $\beta$. For example, consider a choice correspondence $\gamma_{\mathcal{F}}$ over $\mathcal{F}=\{A=\{x, y\}, B=\{x, y, z\}, C=\{y, z\}\}$

\footnotetext{
${ }^{6}$ This property is also known as the Chernoff Axiom. In the context of choice functions, the parallel property is also known as Independence of Irrelevant Alternatives, as already mentioned.

${ }^{7}$ Property $\beta$ : Let $A, B \in \mathcal{F}$ with $A \subseteq B$, if $x, y \in \gamma_{\mathcal{F}}(A)$ and $x \in \gamma_{\mathcal{F}}(B)$, then $y \in \gamma_{\mathcal{F}}(B)$.
} 
such that $A=\gamma_{\mathcal{F}}(A)$ and $x \in \gamma_{\mathcal{F}}(B)$. Notice that, whenever $y \in \gamma_{\mathcal{F}}(C)$, then AC implies that $y \in \gamma_{\mathcal{F}}(B)$. Hence property $\beta$ follows for sets $A$ and $B$. But if in the previous example $y \notin \gamma_{\mathcal{F}}(C)$, then AC has no bite, and hence property $\beta$ could be violated.

It is remarkable how the imposition of these two-stage behavioral procedures on every possible filter is sufficient to characterize any sequentially rationalizable choice, even when the latter requires more than two rationales to be sequentially rationalized.

We can now present the main result of this paper:

Theorem 3.1. Given a choice function $c$, the following two statements are equivalent:

(1) c is sequentially rationalizable.

(2) c satisfies $\mathcal{F}$-consistency.

Proof of Theorem 3.1: $1 \Rightarrow 2$. Suppose that $c$ is sequentially rationalizable by an ordered collection of asymmetric binary relations $\left\{P_{1}, \ldots, P_{K}\right\}$. We have to show that, for every filter $\mathcal{F}$, there is a non-trivial choice correspondence $\gamma_{\mathcal{F}}$ that satisfies $\alpha, \mathrm{AC}$, and is such that $c(A)=c\left(\gamma_{\mathcal{F}}(A)\right)$ for every $A \in \mathcal{F}$.

Let $\mathcal{F}$ be a filter. Consider $\rho=\min \left\{j \in\{1,2, \ldots, K\}\right.$ such that $a P_{j} b$ for some $\{a, b\}$ $\in \mathcal{F}\}$, and define for every $A \in \mathcal{F}, \gamma_{\mathcal{F}}(A)=M_{\rho}(A)$. That is, $P_{\rho}$ is the very first rationale in $\left\{P_{1}, \ldots, P_{K}\right\}$ that applies in $\mathcal{F}$. By construction, it is not difficult to see that for every $A \in \mathcal{F}, \gamma_{\mathcal{F}}(A)=M_{\rho}(A)=M\left(M_{\rho-1}(A), P_{\rho}\right)=M\left(A, P_{\rho}\right)$. We now show that it satisfies the requirements of $\mathcal{F}$-consistency.

- Clearly, by definition of $\rho, \gamma_{\mathcal{F}}$ is a non-trivial choice correspondence on $\mathcal{F}$.

- To show that $\gamma_{\mathcal{F}}$ satisfies $\alpha$, let $A \subseteq B$ with $A, B \in \mathcal{F}$. Let $x \in A \cap \gamma_{\mathcal{F}}(B)$. Then, for every $y \in B \backslash\{x\}$ it cannot be true that $y P_{\rho} x$, and therefore, in particular for every $y \in A \backslash\{x\}$, it cannot be true that $y P_{\rho} x$. Hence, $x \in M_{\rho}(A)=\gamma_{\mathcal{F}}(A)$, as desired.

- To show that $\gamma_{\mathcal{F}}$ satisfies AC, let $x \in \gamma_{\mathcal{F}}(\{x, y\})$ for all $y \in S \backslash\{x\}$ with $S \in \mathcal{F}$. Then, for every $y \in S \backslash\{x\}$, it cannot be true that $y P_{\rho} x$, and trivially it must be the case that $x \in \gamma_{\mathcal{F}}(S)$, as desired.

- Finally, we have to show that $c(A)=c\left(\gamma_{\mathcal{F}}(A)\right)$ for every $A \in \mathcal{F}$. To this end note that, since we are assuming that $c$ is sequentially rationalizable by $\left\{P_{1}, \ldots, P_{K}\right\}$, it must be that $c(A)=M_{K}(A)$ for every choice problem $A$. By definition we have

$$
\begin{aligned}
c(A)=M_{K}(A) & =M_{K}\left(M_{K-1}(A)\right) \\
& =\cdots \\
& =M_{K}\left(M_{K-1}\left(\cdots M_{\rho+1}\left(M_{\rho}(A)\right)\right)\right) .
\end{aligned}
$$


By construction it is the case that $\gamma_{\mathcal{F}}(A)=M_{\rho}(A)$ and therefore we may write

$$
\begin{aligned}
M_{K}\left(M_{K-1}\left(\cdots M_{\rho+1}\left(M_{\rho}(A)\right), \cdots\right)\right) & = \\
M_{K}\left(M_{K-1}\left(\cdots M_{\rho+1}\left(\gamma_{\mathcal{F}}(A)\right), \cdots\right)\right) & = \\
\cdots & = \\
M_{K}\left(\gamma_{\mathcal{F}}(A)\right) & =c\left(\gamma_{\mathcal{F}}(A)\right) .
\end{aligned}
$$

and hence the claim follows.

We have shown that $\gamma_{\mathcal{F}}$ satisfies the requirements and, therefore, $c$ satisfies $\mathcal{F}$ consistency.

$2 \Rightarrow 1$. Assume that $c$ satisfies $\mathcal{F}$-consistency. We first claim the existence of: (i) collections of sets $\left\{\mathcal{F}_{j}\right\}_{j \in \mathbb{N}}$ which are either filters containing all singletons or simply the set of all singletons, (ii) choice correspondences $\left\{\gamma_{\mathcal{F}_{j}}\right\}_{j \in \mathbb{N}}$ over these collections of sets satisfying the properties given by $\mathcal{F}$-consistency and, (iii) rationales $\left\{P_{j}\right\}_{j \in \mathbb{N}}{ }^{8}$

Take $\mathcal{F}_{1}=\mathcal{P}(X)$. It is immediate that $\mathcal{F}_{1}$ is a filter containing all singletons. By $\mathcal{F}$-consistency there is a non-trivial choice correspondence $\gamma_{\mathcal{F}_{1}}$ that satisfies $\alpha, \mathrm{AC}$ and $c(A)=c\left(\gamma_{\mathcal{F}_{1}}(A)\right)$ for every $A \in \mathcal{F}_{1}$. Finally define $P_{1}$ by $a P_{1} b \Leftrightarrow[a \neq b, a=$ $\left.\gamma_{\mathcal{F}_{1}}(\{a, b\})\right]$. It is immediate that $P_{1}$ is asymmetric.

We now prove the inductive step. Suppose that (i) $\left\{\mathcal{F}_{j}\right\}_{j=1}^{t-1}$ is a collection of (collections of) sets which are either filters containing all singletons or the set of all singletons, (ii) $\left\{\gamma_{\mathcal{F}_{j}}\right\}_{j=1}^{t-1}$ is a collection of choice correspondences defined on the corresponding collections of sets, that satisfy the properties given by $\mathcal{F}$-consistency, and (iii) $\left\{P_{j}\right\}_{j=1}^{t-1}$ is a list of rationales. First, define $\mathcal{F}_{t}$ as $\mathcal{F}_{t}=\{A$ : there exists $B \in$ $\mathcal{F}_{t-1}$ such that $\left.A=\gamma_{\mathcal{F}_{t-1}}(B)\right\}$. We check that it is either a filter containing all the singletons or the collection of all singletons. Let $B \in \mathcal{F}_{t}$ and $A \subseteq B$. By definition there is a set $D \in \mathcal{F}_{t-1}$ such that $B=\gamma_{\mathcal{F}_{t-1}}(D)$. Hence, it must be that $A \subseteq B \subseteq D$. Then, since $\mathcal{F}_{t-1}$ is either a filter containing all singletons or the collection of all singletons, it must be that $A \in \mathcal{F}_{t-1}$. Since $A \subseteq D$, by $\alpha, \gamma_{\mathcal{F}_{t-1}}(A) \supseteq A \cap \gamma_{\mathcal{F}_{t-1}}(D)$. But then, $A \cap \gamma_{\mathcal{F}_{t-1}}(D)=A \cap B=A$, which implies that $A=\gamma_{\mathcal{F}_{t-1}}(A)$, and therefore $A \in \mathcal{F}_{t}$, as desired. Hence, if $\mathcal{F}_{t}$ contains a set $S$ with $|S| \geq 2$, then $\mathcal{F}_{t}$ is a filter. Given that $\mathcal{F}_{t-1}$ contains all singletons and $\gamma_{\mathcal{F}_{t-1}}$ is a choice correspondence, the image of each singleton by that correspondence is the singleton itself, and hence all singletons are also in $\mathcal{F}_{t}$ too. This implies that $\mathcal{F}_{t}$ is either a filter containing all singletons or the collection of all singletons.

By $\mathcal{F}$-consistency, if $\mathcal{F}_{t}$ is a filter, there is a non-trivial choice correspondence $\gamma_{\mathcal{F}_{t}}$ that satisfies $\alpha, \mathrm{AC}$, and $c(A)=c\left(\gamma_{\mathcal{F}_{t}}(A)\right)$ for every $A \in \mathcal{F}_{t}$. If $\mathcal{F}_{t}$ is the collection of all singletons, define $\gamma_{\mathcal{F}_{t}}$ as the unique correspondence on $\mathcal{F}_{t}$, i.e., the identity mapping. Finally define $P_{t}$ by $a P_{t} b \Leftrightarrow\left[a \neq b, a=\gamma_{\mathcal{F}_{t}}(\{a, b\})\right]$. It is immediate that $P_{t}$ is asymmetric, and we have concluded the inductive step.

\footnotetext{
${ }^{8}$ As usual, $\mathbb{N}$ denotes the set of strictly positive integers.
} 
We now show that for all $A \in \mathcal{P}(X)$ and for all $j \in \mathbb{N}$, it is the case that $M_{j}(A)=$ $\gamma_{\mathcal{F}_{j}} \circ \gamma_{\mathcal{F}_{j-1}} \circ \cdots \circ \gamma_{\mathcal{F}_{1}}(A) .{ }^{9}$ Consider a set $A \in \mathcal{P}(X)$. We start by proving that $M_{1}(A)=\gamma_{\mathcal{F}_{1}}(A)$. Let $x \in \gamma_{\mathcal{F}_{1}}(A)$. By $\alpha$, we know that $x \in \gamma_{\mathcal{F}_{1}}(\{x, y\})$ for every $y \in A \backslash\{x\}$ and hence, by the definition of $P_{1}$, for every $y \in A \backslash\{x\}$, it is not true that $y P_{1} x$. This shows that, if $x \in \gamma_{\mathcal{F}_{1}}(A)$, then $x \in M_{1}(A)$. Now let $x \in M_{1}(A)$. By definition, for every $y \in A \backslash\{x\}$, it is not true that $y P_{1} x$ and hence it must be that for every $y \in A \backslash\{x\}, x \in \gamma_{\mathcal{F}_{1}}(\{x, y\})$. AC guarantees that $x \in \gamma_{\mathcal{F}_{1}}\left(\bigcup_{y \in A \backslash\{x\}}\{x, y\}\right)=$ $\gamma_{\mathcal{F}_{1}}(A)$, as desired. Consequently, if $x \in M_{1}(A)$ then $x \in \gamma_{\mathcal{F}_{1}}(A)$. We have shown that $M_{1}(A)=\gamma_{\mathcal{F}_{1}}(A)$.

We now prove the inductive step. Suppose that $M_{j}(A)=\gamma_{\mathcal{F}_{j}} \circ \gamma_{\mathcal{F}_{j-1}} \circ \cdots \circ \gamma_{\mathcal{F}_{1}}(A)$ for all $j<t$. We now show that $M_{t}(A)=\gamma_{\mathcal{F}_{t}} \circ \gamma_{\mathcal{F}_{t-1}} \circ \cdots \circ \gamma_{\mathcal{F}_{1}}(A)$. Let it be that $x \in \gamma_{\mathcal{F}_{t}} \circ \gamma_{\mathcal{F}_{t-1}} \circ \cdots \circ \gamma_{\mathcal{F}_{1}}(A)$. By $\alpha, x \in \gamma_{\mathcal{F}_{t}}(\{x, y\})$ for every $y \in \gamma_{\mathcal{F}_{t-1}} \circ \gamma_{\mathcal{F}_{t-2}} \circ$ $\cdots \circ \gamma_{\mathcal{F}_{1}}(A) \backslash\{x\}$. Hence, $y P_{t} x$ for no $y \in \gamma_{\mathcal{F}_{t-1}} \circ \gamma_{\mathcal{F}_{t-2}} \circ \cdots \circ \gamma_{\mathcal{F}_{1}}(A) \backslash\{x\}$ and then, since obviously $x \in \gamma_{\mathcal{F}_{t-1}} \circ \gamma_{\mathcal{F}_{t-2}} \circ \cdots \circ \gamma_{\mathcal{F}_{1}}(A)=M_{t-1}(A)$ we conclude that $x \in M_{t}(A)$, as desired. In the other direction, let $x \in M_{t}(A)$. This implies that $y P_{t} x$ for no $y \in M_{t-1}(A)=\gamma_{\mathcal{F}_{t-1}} \circ \gamma_{\mathcal{F}_{t-2}} \circ \cdots \circ \gamma_{\mathcal{F}_{1}}(A)$, and hence by definition $x \in \gamma_{\mathcal{F}_{t}}(\{x, y\})$ for every $y \in \gamma_{\mathcal{F}_{t-1}} \circ \gamma_{\mathcal{F}_{t-2}} \circ \cdots \circ \gamma_{\mathcal{F}_{1}}(A) \backslash\{x\}$. AC implies that $x \in \gamma_{\mathcal{F}_{t}}\left(\bigcup_{y \in \gamma_{\mathcal{F}_{t-1}} \circ \gamma_{\mathcal{F}_{t-2}} \circ \cdots \circ \gamma_{\mathcal{F}_{1}}(A) \backslash\{x\}}\{x, y\}\right)=\gamma_{\mathcal{F}_{t}} \circ \gamma_{\mathcal{F}_{t-1}} \circ \cdots \circ \gamma_{\mathcal{F}_{1}}(A)$, as desired. Therefore, $M_{t}(A)=\gamma_{\mathcal{F}_{t}} \circ \gamma_{\mathcal{F}_{t-1}} \circ \cdots \circ \gamma_{\mathcal{F}_{1}}(A)$.

We now show that there must exist a value $K \in \mathbb{N}$ such that $\mathcal{F}_{j}$ is a filter if and only if $j \leq K$. Given the construction, it is obvious that $\mathcal{F}_{j+1} \subseteq \mathcal{F}_{j}$ for all $j \in \mathbb{N}$. Therefore, we are left to prove that the collection $\mathcal{F}_{j+1}$ is a strict subset of $\mathcal{F}_{j}$ for $j=1, \ldots K$, and that $\mathcal{F}_{K+1}$ is the collection of singletons. The latter derives from the former given the finiteness assumption. To show that $\mathcal{F}_{j+1} \subset \mathcal{F}_{j}$ for all $j \leq K$, note that whenever $\mathcal{F}_{j}$ is not the collection of all singletons, then it is a filter, and $\gamma_{\mathcal{F}_{j}}$ satisfies non-triviality. Therefore, there exists a set $A \in \mathcal{F}_{j}$ such that $\gamma_{\mathcal{F}_{j}}(A) \neq A$. Take $y \in A$ with $y \notin \gamma_{\mathcal{F}_{j}}(A)$. We claim that $A \notin \mathcal{F}_{j+1}$. By contradiction, suppose $A \in \mathcal{F}_{j+1}$. Then $y \in A=\gamma_{\mathcal{F}_{j}}(B)$ for some $B \in \mathcal{F}_{j}$. But the application of $\alpha$ guarantees that $y \in \gamma_{\mathcal{F}_{j}}(A)$, which is a contradiction. Therefore, filters contract strictly and there must exist a value $K \in \mathbb{N}$ such that $\mathcal{F}_{j}$ is a filter if and only if $j \leq K$.

We finish by proving that $\left\{P_{j}\right\}_{j=1}^{K}$ rationalizes $c$. Given the construction, $\gamma_{\mathcal{F}_{K}} \circ$ $\gamma_{\mathcal{F}_{K-1}} \circ \cdots \circ \gamma_{\mathcal{F}_{1}}(A)$ is a singleton, and then we have that $M_{K}(A)=\gamma_{\mathcal{F}_{K}} \circ \gamma_{\mathcal{F}_{K-1}} \circ$ $\cdots \circ \gamma_{\mathcal{F}_{1}}(A)=c\left(\gamma_{\mathcal{F}_{K}} \circ \gamma_{\mathcal{F}_{K-1}} \circ \cdots \circ \gamma_{\mathcal{F}_{1}}(A)\right)$. Now, since all correspondences satisfy the requirements of $\mathcal{F}$-consistency $c\left(\gamma_{\mathcal{F}_{K}} \circ \gamma_{\mathcal{F}_{K-1}} \circ \cdots \circ \gamma_{\mathcal{F}_{1}}(A)\right)=c\left(\gamma_{\mathcal{F}_{K-1}} \circ \gamma_{\mathcal{F}_{K-2}} \circ\right.$ $\left.\cdots \circ \gamma_{\mathcal{F}_{1}}(A)\right)=\cdots=c\left(\gamma_{\mathcal{F}_{1}}(A)\right)=c(A)$. This shows that the choice is sequentially rationalized. $\square$

\footnotetext{
${ }^{9}$ Where $\circ$ denotes the composition operator of two functions.
} 
Theorem 3.1 shows that, to determine whether a particular choice procedure is sequentially rationalizable, one needs to check for $\mathcal{F}$-consistency. Further, Theorem 3.1 also shows that since $\mathcal{F}$-consistency imply a high degree of behavioral structure, the notion of sequential rationalizability is a tight one.

\section{Applications}

Our characterization result allows to identify what is and what is not sequentially rationalizable. This, in turn, opens up the possibility of judging whether choice models that may have little to do, in principle, with sequential rationalizability, are in fact connected to the notion of sequential rationalizability. In this section, we illustrate these new possibilities by first establishing a perhaps unexpected relation between sequential rationalizability and another important recent notion of rationalizability: rationalizability by game trees. We then show that agenda rationalizability, a notion that is rooted in individual and social procedures of selection through the ordered elimination of alternatives, is also sequentially rationalizable.

It is convenient to mention, in advance, the connection between sequential rationalizability and two polar notions of rationalizability. On the one hand, it is immediate that every standard rationalizable function ${ }^{10}$ is sequentially rationalizable, while the reverse does not hold. On the other hand, every choice function, and thus every sequentially rationalizable choice function, is rationalizable by multiple rationales á la Kalai, Rubinstein and Spiegler (2002), but, again, the converse does not hold.

4.1. Rationalizability by Game Trees. In this section we show that any choice function that is rationalizable by game trees is in fact sequentially rationalizable. As the converse does not hold, the set of choice functions rationalizable by a game tree is a strict subset of the set of choice functions satisfying sequential rationalizability.

$\mathrm{Xu}$ and Zhou characterize those choice functions that can be rationalized by extensive games with perfect information. For a choice function $c$ to be rationalizable by game trees, there must be a tree $G$ that: (i) has alternatives $X$ as terminal nodes, such that each alternative in $X$ appears once and only once as a terminal node of $G$ (hence, $X$ and $G$ can be identified), and (ii) every node in the tree $G$ must represent a decision by an agent $i$, with a linear ordering $R_{i}$ over $X$.

Rationalizability by Game Trees: A choice function $c$ is rationalizable by game trees whenever there exists a game tree $G$ such that

$$
c(A)=S P N E(G \mid A ; R) \text { for all } A \in \mathcal{P}(X),
$$

where $G \mid A$ is the reduced tree of $G$ that retains all the branches of $G$ leading to terminal nodes in $A, R$ is the linear order profile, and $S P N E(\Gamma)$ stands for the subgame

\footnotetext{
${ }^{10}$ That is, every choice function that satisfies IIA.
} 
perfect Nash equilibrium outcome of $\Gamma$.

Denote by $\mathcal{C}^{S R}$ and $\mathcal{C}^{R G T}$ the sets of choice functions that are, respectively, sequentially rationalizable and rationalizable by game trees. We can now present the following result.

Theorem 4.1. $\mathcal{C}^{R G T} \subset \mathcal{C}^{S R}$.

Proof of Theorem 4.1: We show that $\mathcal{C}^{R G T} \subseteq \mathcal{C}^{S R}$ by way of $\mathcal{F}$-consistency. We first need the following definitions. Given a filter $\mathcal{F}$ a node $i$ is relevant for $\mathcal{F}$ if there is a set $A$ in $\mathcal{F}$, with $|A| \geq 2$, such that for all $x \in A, x$ is a successor of $i$. We say that $i$ is minimally relevant if it is relevant and no successor of $i$ is relevant. That is, $i$ is minimally relevant whenever $i$ decides the final choice among a set $A$ in $\mathcal{F}$.

Given a filter $\mathcal{F}$ select a node $i$ that is is minimally relevant. Then, there is a set $A$ in $\mathcal{F}$, with $|A| \geq 2$, where $i$ makes the final choice. Pick the worst alternative in this set according to $R_{i}$, and denote it by $y$. Take any other alternative $x \in A \backslash\{y\}$ and let

$$
\gamma_{\mathcal{F}}(A)=\left\{\begin{array}{l}
A \text { if } x \notin A \text { or } y \notin A \\
A \backslash\{y\} \text { if } x, y \in A
\end{array}\right.
$$

It is immediate that $\gamma_{\mathcal{F}}$ is non-trivial, satisfies $\mathrm{AC}$, and $\alpha$. We have to show that $c(A)=c\left(\gamma_{\mathcal{F}}(A)\right)$ for every $A$ in $\mathcal{F}$. Given the definition of $\gamma_{\mathcal{F}}(A)$ we only need to show the latter equality for cases in which $x$ and $y$ are elements of $A$. But, since, by assumption, $c(A)=S P N E(G \mid A ; R)$ and $i$ is a terminal decision in which $y$ is dominated, backward induction guarantees that $c(A)=S P N E(G \mid A ; R)=$ $\operatorname{SPNE}(G \mid(A \backslash\{y\}) ; R)=c(A \backslash\{y\})=c\left(\gamma_{\mathcal{F}}(A)\right)$. Therefore, $\mathcal{F}$-consistency holds, and then Theorem 3.1 guarantees that $\mathcal{C}^{R G T} \subseteq \mathcal{C}^{S R}$.

We show, by way of an example, that $\mathcal{C}^{S R} \nsubseteq \mathcal{C}^{R G T}$. Let $c\left(x_{1}, x_{2}, y_{1}\right)=y_{1}, c\left(x_{1}, x_{2}, y_{2}\right)=$ $x_{2}, c\left(x_{1}, y_{1}, y_{2}\right)=x_{1}, c\left(x_{2}, y_{1}, y_{2}\right)=y_{1}, c\left(x_{1}, x_{2}, y_{1}, y_{2}\right)=y_{1}, c\left(x_{1}, x_{2}\right)=x_{2}, c\left(x_{1}, y_{1}\right)=$ $x_{1}, c\left(x_{1}, y_{2}\right)=y_{2}, c\left(x_{2}, y_{1}\right)=y_{1}, c\left(x_{2}, y_{2}\right)=x_{2}$, and $c\left(y_{1}, y_{2}\right)=y_{1}$.

To see that $c \notin \mathcal{C}^{R G T}$, we show that $c$ does not satisfy the property divergence consistency, which $\mathrm{Xu}$ and Zhou show to be a necessary condition for rationalizability by game trees. First, consider the following definitions. For any triple $x, y, z, x$ diverges before $y$ and $z$, if $c(\{x, y\})=x, c(\{y, z\})=y$, and $c(\{z, x\})=z$ (or a similar condition holds for some permutation of $x, y$, and $z)$, and $c(\{x, y, z\})=x$. Finally, divergence consistency states that, for any four alternatives $x_{1}, x_{2}, y_{1}, y_{2}$, if $x_{1}$ diverges before $y_{1}$ and $y_{2}$, and $y_{1}$ diverges before $x_{1}$ and $x_{2}$, then $c\left(\left\{x_{1}, y_{1}\right\}\right)=x_{1}$ if and only if $c\left(\left\{x_{2}, y_{2}\right\}\right)=y_{2}$.

It can be easily seen that, in our example, $x_{1}$ diverges before $y_{1}$ and $y_{2}$, and $y_{1}$ diverges before $x_{1}$ and $x_{2}$. At the same time, we have $c\left(x_{1}, y_{1}\right)=x_{1}$ but $c\left(x_{2}, y_{2}\right)=$ 
$x_{2}$, contradicting divergence consistency. However, the choice function is sequentially rationalizable by the rationales: $P_{1}=\left\{\left(y_{1}, y_{2}\right)\right\}, P_{2}=\left\{\left(x_{2}, x_{1}\right)\right\}$ and $P_{3}=$ $\left\{\left(x_{1}, y_{1}\right),\left(y_{2}, x_{1}\right),\left(y_{1}, x_{2}\right),\left(x_{2}, y_{2}\right)\right\}$. This ends the proof of the theorem. $\square$

The above proof puts in action our property, $\mathcal{F}$-consistency, to show that a choice function that is rationalizable by game trees is also sequentially rationalizable.

4.2. Agenda Rationalizability. We now introduce a rationalizability notion that is rooted in (i) a particular choice procedure that is attracting some attention in the economics and psychology literature, and (ii) in a class of voting mechanisms. Let us first introduce the details and then draw the connections with the above two strands of literature.

Let us assume that the $n$ elements in $X$ are linearly ordered $1<2<\cdots<n$. This order may be interpreted as, say, a particular physical presentation of the objects. For any choice problem $A$ in $X$, write the elements in $A$ ordered according to < by $a(1)<a(2)<\cdots<a(t)$. The DM makes a selection from $A$ according to the following elimination process. First she makes a first selection between $a(1)$ and $a(2)$, then compares the selected element from $a(1), a(2)$ with $a(3)$ and makes a new selection. The DM continues in this ordered way until the surviving element is compared with the last element $a(t)$; this last choice determines the choice in $A$. Let that whenever two alternatives $x, y$ are compared, the selection between $x$ and $y$ is the same, irrespective of the set $A$ to which $x$ and $y$ belong.

Similar choice by ordered elimination procedures are studied in the choice-theoretic literature. The models studied in Rubinstein and Salant (2006) and Salant and Rubinstein (2008), for example, include this one as a special case. See also Masatlioglu and Nakajima (2007). But the binary choices between alternatives may also be the result of majority voting, for example. Then voting mechanisms like voting by successive elimination are also connected to the above (see Dutta, Jackson, and Le Breton, 2002).

We can now introduce the following notion of rationalizability.

Agenda Rationalizability: A choice function $c$ is agenda rationalizable whenever there exists an order $<$ over the set of alternatives (an agenda) and binary choices such that for every $A \in \mathcal{P}(X), c$ is the outcome of the elimination process.

McGarvey (1953) strengthens the connection between agenda rationalizability and voting. McGarvey (1953) already showed that, for every possible connected asymmetric relation over the set of alternatives, there is a set of individuals with linear orders over the set of alternatives such that majority voting over pairs of alternatives determine the connected asymmetric relation. 
Here we wonder about the relation between agenda rationalizability and sequential rationalizability. Denote by $\mathcal{C}^{A R}$ the set of choice functions that are agenda rationalizable. We can now present the following result.

Theorem 4.2. $\mathcal{C}^{A R} \subset \mathcal{C}^{S R}$.

Proof of Theorem 4.2: Theorem 3.1 tells us that we simply have to check whether $\mathcal{F}$-consistency holds. But this is straightforward. For any filter $\mathcal{F}$ let $x$ be the first alternative according to $<$ such that there is another alternative $z \in X \backslash\{x\}$ with $\{x, z\}$ in $\mathcal{F}$. Now, given $x$, select the pair $\{x, y\}$ in $\mathcal{F}$ such that there is no $z$ with $z<y$ and $\{x, z\}$ in $\mathcal{F}$. That is, $\{x, y\}$ is lexicographically the first pair in $\mathcal{F}$ according to $<$. Assume, without loss of generality, that $x=c(\{x, y\})$. Then, define, as in Theorem 4.1

$$
\gamma_{\mathcal{F}}(A)=\left\{\begin{array}{l}
A \text { if } x \notin A \text { or } y \notin A \\
A \backslash\{y\} \text { if } x, y \in A
\end{array}\right.
$$

It is immediate that $\gamma_{\mathcal{F}}$ is non-trivial, satisfies AC, $\alpha$, and that $c(A)=c\left(\gamma_{\mathcal{F}}(A)\right)$ for every $A$ in $\mathcal{F}$. Hence, $\mathcal{F}$-consistency holds and Theorem 3.1 implies that $\mathcal{C}^{A R} \subseteq \mathcal{C}^{S R}$. The example in the proof of Theorem 4.1 can be used to show that in fact $\mathcal{C}^{A R} \neq$ $\mathcal{C}^{S R}{ }^{11} \square$

Like in the case of rationalizability by game trees, we have used $\mathcal{F}$-consistency to establish the relation between agenda and sequential rationalizability. This simple proof illustrates the usefulness of $\mathcal{F}$-consistency.

\section{Final Remarks}

In this paper we have studied the structure of sequential rationalizability. In essence, we contribute to the literature in two different ways. First, we clarify the behavioral structure of sequential rationalizability. We do so by offering a characterization result, in which we prove that a testable property, $\mathcal{F}$-consistency, is equivalent to the notion of sequential rationalizability.

Second, we show that our characterization result is useful in the sense that it facilitates investigation of the relationships between different notions of rationalizability, and it allows verification of whether particular choice procedures are sequentially rationalizable. We have shown that the notions of rationalizability by game trees and agenda rationalizability are both strict refinements of sequential rationalizability.

\footnotetext{
${ }^{11}$ Indeed, it can be shown that every agenda rationalizable choice function is rationalizable by game trees. We can provide details upon request.
} 
This suggests that sequential rationalizability may be of interest in a wider range of environments that perhaps initially expected.

\section{REFERENCES}

[1] Dutta, B., M. Jackson, and M. Le Breton (2002), "Voting by Successive Elimination and Strategic Candidacy," Journal of Economic Theory, 103:190-218.

[2] Kalai, G., A. Rubinstein, and R. Spiegler (2002), "Rationalizing Choice Functions by Multiple Rationales," Econometrica, 70:2481-88.

[3] Manzini, P. and M. Mariotti (2007), "Sequentially Rationalizable Choice," American Economic Review, 97:1824-1839.

[4] Masatlioglu, Y. and D. Nakajima (2007), "A Theory of Choice by Elimination," mimeo, University of Michigan.

[5] McGarvey, D.C. (1953), "A Theorem on the Construction of Voting Paradoxes," Econometrica, 21:608-610.

[6] Rubinstein, A. and Y. Salant (2006), "A Model of Choice from Lists," Theoretical Economics, $1: 3-17$.

[7] Salant, Y. and A. Rubinstein (2008), “( $A, f)$ : Choice with Frames," Review of Economic Studies, fortcoming.

[8] Xu, Y. and L. Zhou (2007) "Rationalizability of Choice Functions by Game Trees," Journal of Economic Theory, 134:548-556. 